NYU-TH/00/01/02

CERN-TH/2000-041

Bicocca-FT/00/04

hep-th/0002066

\title{
A NOTE ON THE HOLOGRAPHIC BETA AND $C$ FUNCTIONS
}

\author{
D. Anselmi ${ }^{a}$, L. Girardello $^{b, d}$, M. Porrati $^{c}$ and A. Zaffaroni ${ }^{d}$ \\ ${ }^{a}$ CERN, Division Théorique, CH-1211, Geneva 23, Switzerland \\ ${ }^{b}$ Università di Milano-Bicocca, Dipartimento di Fisica \\ c Department of Physics, NYU, 4 Washington Pl, New York NY 10012 \\ ${ }^{d}$ INFN - Sezione di Milano, Via Celoria 16, Milan, Italy
}

\begin{abstract}
The holographic RG flow in AdS/CFT correspondence naturally defines a holographic scheme in which the central charge $c$ and the beta function are related by the formula $\dot{c}=-2 c \beta_{a} \beta_{b} G^{a b}$, where $G^{a b}$ is the metric of the kinetic term of the supergravity scalars. In particular, the metric in the space of couplings is $f^{a b}=2 c G^{a b}$. We perform some checks of that result and we compare it with the quantum field theory expectations. We discuss alternative definitions of the $c$-function. In particular, we compare, for a particular supersymmetric flow, the holographic $c$-function with the central charge computed directly from the two-point function of the stressenergy tensor.
\end{abstract}


Conformal field theories in four dimensions have two main central charges, $c$ and $a$, which multiply the square of the Weyl tensor and the Euler density, respectively, in the trace anomaly. In a quantum field theory interpolating between UV and IR conformal fixed points the total flows of $c$ and $a$, i.e. the differences $\Delta c=c_{\mathrm{UV}}-c_{\mathrm{IR}}$ and $\Delta a=a_{\mathrm{UV}}-a_{\mathrm{IR}}$, give important physical information (see for instance 《1). The flows can be induced by dimensionful parameters, by the renormalization-group scale $\mu$, or by the combined effect of both.

The RG flow, induced by $\mu$, is irreversible, which means that it satisfies the inequality $\Delta a \geq 0$. The irreversibility of the $\mathrm{RG}$ flow is better studied when dimensionful parameters are absent. This means that the theory is conformal at the classical level. In [2] a nonperturbative formula for the RG flow $\Delta a$ was obtained and checked in perturbation theory.

The flows induced by relevant deformations have a quantitatively different effect on $\Delta a$, although they still obey the inequality $\Delta a \geq 0$. Nevertheless, there is a special class of theories in every even dimension, the theories interpolating between $c=a$ fixed points, where the formula for $\Delta a$ (equal to $\Delta c$ ) is universal [3]. This universality also holds in two dimensions. The even-dimensional conformal field theories with $c=a$ share various properties with two dimensional conformal field theory [3].

The "holographic" supergravity/gauge theory correspondence considers, in the 5-d gauged supergravity limit, precisely a class of $c=a$ conformal field theories 四, the simplest example being the $\mathrm{N}=4$ supersymmetric Yang-Mills theory in the strongly coupled large- $N$ limit. Other examples have been constructed in the literature and need not be supersymmetric.

In [5, 6, 7, 8, 9, 10] flows induced by massive deformations were considered in the context of this correspondence. We call them the "holographic" flows. On the basis of the considerations recalled above, we expect, and are indeed going to check in the present paper, that:

1) The "holographic" c-function defined in [5] obeys a formula similar to the formula for the RG flow of the a-function in quantum field theory. This is our result (8). In particular, the holographic central charge $c$ is stationary at the fixed points. Observe that the stationarity of $c$ is not true in a general quantum field theory (with $c \neq a$ at the fixed points) and is peculiar of the holographic flows;

2) Other definitions of $c$, not related to the equality $c=a$, but equally convenient in quantum field theory, for example the central function defined by the stress-tensor two-point function, should exhibit similar properties: monotonicity and stationarity at the fixed points. These facts are also peculiar of the holographic flows, because it is well known that $c$ does not even decrease in a general quantum field theory.

We begin by discussing the properties of the $c$-function proposed in ref. [5] and work 
out the general formula for its derivative $\dot{c}$ along the flow. Secondly, we directly compute the $c$-function using the correlator of two stress-energy tensors, always using the holographic correspondence, and compare the two definitions. We explain why the two definitions are compatible (in particular, both positive and interpolating monotonically between the critical values) even though they are not equal.

A candidate $c$-function, decreasing along the holographic flow was proposed in [5, 9]. In the notation of [5] the $c$-function is:

$$
c=\text { const. }\left(T_{y y}\right)^{-3 / 2}=\left(\frac{\mathrm{d} \phi}{\mathrm{d} y}\right)^{-3},
$$

Where $\phi$ is the scale factor of the 5 -d supergravity metric, and $y$ is its radial coordinate: $d s^{2}=d y^{2}+\exp (2 \phi) d x_{\mu} d x^{\mu}$.

The equations for a holographic RG flow generated by one of the perturbations that can be studied within 5 -d gauged supergravity are, in the notations of ref. [0] ${ }^{1}$ :

$$
\frac{\mathrm{D}}{\mathrm{D} y}\left(e^{4 \phi} G_{a b} \frac{\mathrm{d} \lambda^{b}}{\mathrm{~d} y}\right)=e^{4 \phi} \frac{\partial V}{\partial \lambda^{a}}, \quad 6\left(\frac{\mathrm{d} \phi}{\mathrm{d} y}\right)^{2}=\sum_{a b} G_{a b} \frac{\mathrm{d} \lambda^{a}}{\mathrm{~d} y} \frac{\mathrm{d} \lambda^{b}}{\mathrm{~d} y}-2 V .
$$

Here $\lambda^{a}$ denotes the 42 scalars of 5 - $\mathrm{d} N=8$ gauged supergravity, $G_{a b}$ denotes the metric of their kinetic term and D/Dy is the covariant derivative. From now on we will set for simplicity and with no loss of generality $G_{a b}=\delta_{a b}$.

These equations imply, in particular, that the second derivative of $\phi$ does not depend on the potential $V$ :

$$
\frac{\mathrm{d}^{2} \phi}{\mathrm{d} y^{2}}=-\frac{2}{3} \sum_{a}\left(\frac{\mathrm{d} \lambda_{a}}{\mathrm{~d} y}\right)^{2}
$$

We also have

$$
\frac{\mathrm{d} c}{\mathrm{~d} \phi}=-3\left(\frac{\mathrm{d} \phi}{\mathrm{d} y}\right)^{-5} \frac{\mathrm{d}^{2} \phi}{\mathrm{d} y^{2}}=2 c \sum_{a}\left(\frac{\mathrm{d} \lambda_{a}}{\mathrm{~d} \phi}\right)^{2} .
$$

To obtain quantitative agreement with QFT results (see [2]) and a consistent picture of the holographic RG flow we must set

$$
\phi=\ln \mu, \quad \beta_{a}=\frac{\mathrm{d} \lambda_{a}}{\mathrm{~d} \phi}
$$

Therefore:

$$
\dot{c}=-\frac{\mathrm{d} c}{\mathrm{~d} \phi}=-2 c \sum_{a} \beta_{a}^{2} .
$$

1 These perturbations have UV dimension 2 or 3 ; in gauge theory, they correspond to mass terms for scalars and/or fermions, and trilinear terms in the scalar potential. In supergravity, they correspond to VEVs of some of the 42 scalars in the 5 -d, $\mathrm{N}=8$ supergravity multiplet. 
Let us recall a few other results from quantum field theory [11]. Defining

$$
\Theta=\beta_{a} \mathcal{O}_{a}
$$

and

$$
\mu \frac{\mathrm{d}}{\mathrm{d} \mu} \beta_{a}=-\dot{\beta}_{a}=\Delta_{a b} \beta_{b},
$$

a theorem proved in [11] states that the critical value $h_{*}$ of the $\Theta$-anomalous dimension,

$$
\langle\Theta(x) \Theta(0)\rangle=\frac{\text { const. }}{|x|^{8+2 h_{*}}},
$$

equals the minimal real part of the $\Delta$-eigenvalues, in the IR limit, and the maximal real part of the $\Delta$-eigenvalues in the UV limit. Note that $h_{*}$ is also the anomalous dimension of the off-critical deformation of the theory, i.e. the operator $\lambda_{a} \mathcal{O}_{a}$ (the deformation being $\mathcal{L}_{*} \rightarrow \mathcal{L}=\mathcal{L}_{*}+\lambda_{a} \mathcal{O}_{a}$, where $\mathcal{L}_{*}$ denotes the critical Lagrangian).

Formula (四) implies, in particular,

$$
-\frac{\ddot{c}}{2 \dot{c}}=\frac{\sum_{a, b} \beta_{a} \Delta_{a b} \beta_{b}}{\sum_{a} \beta_{a}^{2}}+\sum_{a} \beta_{a}^{2} .
$$

At criticality the second term vanishes, while the first term selects the minimal- or maximal-real-part eigenvalue of the matrix $\Delta_{a b}$, as we now show. Note that $\Delta_{a b}$ is in general not symmetric. We can diagonalize it in a complex space. Let $\Delta=P^{-1} D P$, with $D=\operatorname{diag}\left(\delta_{a}\right), \delta_{a}$ denoting the eigenvalues. Let us write, around the critical point,

$$
\beta_{a}(\lambda)=\Delta_{a b} \lambda_{b}, \quad \beta_{a}(\mu)=\Delta_{a b} \mu^{\Delta_{b c}} k_{c}=\left(P^{-1} D \mu^{D} P k\right)_{a},
$$

$k_{c}$ denoting arbitrary constants. Now, in the UV limit $(\mu \rightarrow \infty)$ the behavior of the first term of (6) is dominated by the eigenvalue of the matrix $\Delta_{a b}$ with maximal real part. It is dominated by the eigenvalue with minimal real part in the IR limit $(\mu \rightarrow 0)$. The imaginary parts of the eigenvalues are irrelevant phases. In conclusion, we have

$$
-\frac{\ddot{c}}{2 \dot{c}}=\max \operatorname{Re} \delta_{a} \quad \text { in the UV, } \quad-\frac{\ddot{c}}{2 \dot{c}}=\min \operatorname{Re} \delta_{a} \quad \text { in the IR. }
$$

These are also the values of the anomalous dimensions of the operators $\mathcal{O}_{a}$ at criticality, as proved in [11]. Therefore we have, in complete generality,

$$
h_{*}=-\lim _{*} \frac{\ddot{c}}{2 \dot{c}},
$$

where the star denotes criticality. 
The "anomalous dimension" $h_{*}$ denotes the deviation of the total dimension from the reference value $4, h_{*}=\Delta-4$ in the conventional notation. We can check, in complete generality, that this quantum field theoretical prediction is correctly reproduced by the holographic flows. Indeed, the second equation of (2) implies that, around a fixed point,

$$
\frac{\mathrm{d} \phi}{\mathrm{d} y}=\frac{1}{R},
$$

$R$ being the AdS radius, and the first of eqs. (2) gives

$$
\lambda \sim \text { const. } \mathrm{e}^{-(4-\Delta) y / R}=\text { const. } \mathrm{e}^{-(4-\Delta) \phi}
$$

At this point, it is straightforward to see that (7) gives $\Delta-4$. The same can be see from the definition of $\beta$ in (31), confirming that the natural definition of holographic beta function works correctly.

All the results described above generalize to non-canonical scalar metrics, in particular

$$
\dot{c}=-2 c G^{a b} \beta_{a} \beta_{b} .
$$

We must remark that our definition of $c$ is unique only at the critical points $\dot{c}=0$. Away from criticality, $c$ need not coincide with central functions defined in other ways; indeed, it need not coincide with other holographic definitions of $c$, as for instance that given in ref. [13]. This non-uniqueness even within the holographic scheme follows from the ambiguity in the identification of $\phi$ as a function of the scale $\mu$. Only at the critical points, $\dot{c}=0$, is the standard identification, $\phi=\log \left(\mu / \mu_{0}\right)$, unique, because of the AdS/CFT correspondence. Away from criticality, uniqueness is lost.

A canonical definition of $c$ as a function of the scale is obtained by computing the two point function of the stress-energy tensor using the equation [12

$$
\left\langle T_{\mu \nu}(x) T_{\rho \sigma}(0)\right\rangle=-\frac{1}{48 \pi^{4}} \prod_{\mu \nu \rho \sigma}^{(2)}\left[\frac{c(x)}{x^{4}}\right]+\pi_{\mu \nu} \pi_{\rho \sigma}\left[\frac{f(x)}{x^{4}}\right],
$$

where $\pi_{\mu \nu}=\partial_{\mu} \partial_{\nu}-\eta_{\mu \nu} \partial^{2}$, and $\prod_{\mu \nu \rho \sigma}^{(2)}=2 \pi_{\mu \nu} \pi_{\rho \sigma}-3\left(\pi_{\mu \rho} \pi_{\nu \sigma}+\pi_{\mu \sigma} \pi_{\nu \rho}\right)$. We will call this $c$ the canonical $c$-function.

For a generic flow, it is impossible to compute analytically this two-point function, even in the supergravity approximation. To the best of our knowledge, there are few exceptions, namely, the solutions describing the Coulomb branch of the $\mathrm{N}=4$ supersymmetric gauge theory [10] and the $\mathrm{N}=1$ supersymmetric flows studied in ref. [7], which interpolate between the $\mathrm{N}=4 \mathrm{UV}$ theory and an IR N=1 pure super Yang-Mills theory.

Here, we mostly consider the flow to pure $\mathrm{N}=1 \mathrm{YM}$ theory. Only a few modifications of the computation described below are required to study the $\mathrm{N}=4$ Coulomb branch, which will be briefly discussed at the end of this paper. 
The flow we shall consider corresponds to an IR vacuum with zero gaugino condensate. By rescaling the AdS radius to $R=1$ and setting the IR singularity of the metric at $y=0$ (see ref. [7] for details), the 5 -d metric is completely specified by the scale factor

$$
e^{2 \phi(y)}=e^{2 y}-1
$$

To compute the two-point function of the transverse-traceless part of the stress-energy tensor using the holographic correspondence, we need to solve the linearized equations of motion for the 5 -d graviton on the background specified by eq. (10). These equations simplify dramatically for the transverse-traceless part, when they become identical with the equations of motion of a minimally-coupled massless scalar, denoted here by $\chi(x)$. By writing $\chi(x)=\exp \left(i k_{\mu} x^{\mu}\right) \chi_{k}$ we find

$$
-\frac{\mathrm{d}^{2}}{d y^{2}} \chi_{k}-4 \frac{d \phi}{d y} \frac{\mathrm{d}}{d y} \chi_{k}+k^{2} e^{-2 \phi} \chi_{k}=0 .
$$

With the change of variable $x=\exp (-2 y)$, a few elementary algebraic manipulations, and dropping the label $k$, eq. (11) reduces to a standard hypergeometric equation

$$
x(1-x) \frac{\mathrm{d}^{2}}{d y^{2}} \chi-(1+x) \frac{\mathrm{d}}{d y} \chi+a^{2} \chi=0, \quad a^{2} \equiv-\frac{k^{2}}{4},
$$

whose two solutions are (cfr. [14 for notations)

$$
\begin{aligned}
\chi_{1}= & x^{2} F(a+2,-a+2 ; 3 ; x), \\
\chi_{2}= & x^{2} \log (x) F(a+2,-a+2 ; 3 ; x)+\sum_{n=1}^{\infty}[\psi(a+n+2)-\psi(a+2)+\psi(-a+n+2)+ \\
& -\psi(-a+2)-\psi(n+3)+\psi(3)-\psi(n+1)+\psi(1)] \frac{(a+2)_{n}(-a+2)_{n}}{3_{n} n !} x^{n+2}+ \\
& +\frac{4}{a^{2}\left(a^{2}-1\right)}-x .
\end{aligned}
$$

The linear combination of $\chi_{1}, \chi_{2}$ regular at $x=1$ and normalized to 1 at $x=0$ is

$$
\chi=\frac{a^{2}\left(a^{2}-1\right)}{4}\left\{\chi_{2}-[2-\psi(a+2)-\psi(-a+2)+\psi(3)+\psi(1)] \chi_{1}\right\} .
$$

The two-point function of the stress-energy tensor is extracted from this expression in the usual manner [15, 16]. Namely, we compare eq. (15) with eq. (9), and we normalize the central charge in the UV using formula (32) of ref. [15]. To simplify, we choose as in [15] a Euclidean 4 -momentum $\left(k^{2} \geq 0\right)$ oriented along the $z$ coordinate, and we find

$$
\left\langle\tilde{T}_{x y}(k) T_{x y}(0)\right\rangle=-\frac{N^{2}}{64 \pi^{2}} k^{2}\left(k^{2}+4\right) \operatorname{Re} \psi(2+i k)+P\left(k^{2}\right) .
$$


Here $P\left(k^{2}\right)$ denotes a polynomial in $k^{2}$ which only contributes to contact terms. In the UV this formula approaches, obviously, the pure-AdS form

$$
\left\langle\tilde{T}_{x y}(k) T_{x y}(0)\right\rangle=-\frac{N^{2}}{64 \pi^{2}} k^{4} \log \left(k^{2}\right)+\tilde{P}\left(k^{2}\right) .
$$

The 2-point function of the transverse-traceless part of $T_{\mu \nu}$ is proportional to eq. (16), as we noticed above. From equation (9), we can read the $c$-function

$$
\int d^{4} x e^{i k x} \frac{c(x)}{|x|^{4}}=-\frac{\pi^{2} N^{2}}{4} \frac{k^{2}+4}{k^{2}} \operatorname{Re} \psi\left(2+\frac{i k}{2}\right)=-\frac{\pi^{2} N^{2}}{4} \sum_{n=2}^{\infty} \frac{k^{2}+4}{n\left(4 n^{2}+k^{2}\right)}
$$

where we used the series expansion for $\psi$. In the right hand side of this equation, we discarded any contact or trace term.

The Fourier transform can be inverted (modulo contact terms) to give a closed expression for $c(x)$

$$
c(x)=\frac{N^{2}}{2} \sum_{n=2}^{\infty}\left(n^{2}-1\right)|x|^{3} K_{1}(2 n|x|)=\frac{N^{2}}{2}|x|^{3} \int_{0}^{\infty} \frac{3 e^{2 x \cosh t}-1}{\left(e^{2 x \cosh t}-1\right)^{3}} \cosh t d t
$$

Every candidate $c$-function has to satisfy some crucial requirements. First of all, it must be positive definite; this is manifest from equation (19). Second, it must coincide with the value of the central charge at the fixed points of the RG group. This is indeed the case. For small $x, c(x) \rightarrow c_{U V}=N^{2} / 8$, while for large $x, c(x) \sim x^{5 / 2} e^{-4 x} \rightarrow c_{I R}=0$, as appropriate for a confining theory. Finally, it must be monotonic. This can be checked by an explicit computation:

$$
\dot{c}=x \frac{d c}{d x}=\frac{3 N^{2} x^{3}}{2} \int_{0}^{\infty} \frac{\left(3 e^{2 x \cosh t}-1\right)\left(e^{2 x \cosh t}-1\right)-4 x \cosh t e^{4 x \cosh t}}{\left(e^{2 x \cosh t}-1\right)^{4}} \cosh t d t
$$

One can easily check that the integrand is negative definite. This is a non-trivial result that confirms the interpretation of supergravity solutions as description of quantum field theory RG flows.

We can compute the first terms in the small $x$-expansion of $c(x)$

$$
c(x)=N^{2}\left[\frac{1}{8}+\frac{x^{2}}{4} \log x+O\left(x^{2}\right)\right]
$$

Inserting this expansion into formula (7) we find correctly $h_{\mathrm{UV}}=-1$, since the deformation is generated by a fermionic mass term $(\Delta=3)$.

The holographic $c$-function for the flow to pure $\mathrm{N}=1 \mathrm{YM}$ is easily computed from equation (10). With the naive identification $\phi=\log \left(\mu / \mu_{0}\right), \mu_{0}=$ constant, one finds

$$
c_{\mathrm{H}}(\mu)=\frac{N^{2}}{8} \frac{\mu^{6}}{\left(\mu^{2}+\mu_{0}^{2}\right)^{3}}
$$


The $c$-function given by eq. (9), instead, depends non-analytically on $\mu \propto 1 / x$ already at small $x$, as shown by eqs. (21) and (22). This result does not mean that the two definitions are incompatible, rather, as pointed out before, it means that the identification of $\exp (\phi)$ with the scale $\mu$ does not hold outside the critical points.

The computation of the function $f(x)$ in eq. (9) would be interesting because, as noticed in ref. [2], it is related to the derivative of $c(x)$. Unfortunately, the computation of $f(x)$ can not be reduced to the one for a minimally coupled scalar field and requires the full stress-energy tensor two point function.

We conclude with a few observations about the holographic scheme.

i) An ansatz such as $\dot{c} \sim \beta^{k}$ with $k \neq 2$ would disagree with the AdS/CFT correspondence (it would not verify the check above). It would disagree also with quantum field theory [2]. In this sense we have a consistent check of holography versus quantum field theory.

ii) The metric $f$ in the space of couplings [2] - i.e. the higher dimensional analogue of the Zamolodchikov metric - is the metric of the 5-d supergravity scalars times $c$ itself:

$$
f_{a b}=2 c G_{a b} .
$$

Therefore, the monotonicity of $c$ is directly implied by the positivity of $c$ and $G_{a b}$, and vice versa. This is not completely surprising, because we can expect the metric $f$ to be related to the normalization of the two-point functions of scalar operators. From 5d supergravity,

$$
S=\int \sqrt{g}\left(-\frac{R}{4}+G_{a b} \partial \lambda^{a} \partial \lambda^{b}\right)
$$

we can see by a simple scaling that, at least at the fixed points, where $d s^{2}=R^{2}\left[d y^{2}+\right.$ $\left.\exp (2 y) \sum_{i} d x_{i}^{2}\right]$

$$
\begin{gathered}
\langle T(x) T(0)\rangle=\frac{c}{|x|^{8}} \quad \rightarrow \quad c \sim R^{3} \sim(\Lambda)^{-3 / 2} \\
\left\langle\lambda_{a}(x) \lambda_{b}(0)\right\rangle=\frac{f_{a b}}{|x|^{2 \Delta}} \quad \rightarrow \quad f_{a b} \sim R^{3} G_{a b} \sim c G_{a b}
\end{gathered}
$$

The first equation reproduces the known result for $c$ [4], the second one confirms equation (23).

iii) Using the arguments of [2] it is straightforward to show that (23) defines a consistent scheme choice, at least when $c_{\mathrm{IR}} \neq 0$. We call this scheme the "holographic scheme" and can be considered in the class of "proper" schemes of [2], in which the metric $f$ is set equal to a known, positive function: the identity in [2], $2 c G^{a b}$ here. The choice $f_{a b}=\delta_{a b}$ defines the proper beta function $\beta_{\mathrm{P}}$ and relates the total $c$-flow to the area of the graph of the beta function. In the holographic scheme, instead, we have (for $G^{a b}=\delta^{a b}$ ), $f^{a b}=2 c \delta^{a b}$, i.e. the total flow of $\ln c$ is (twice) the area of the graph of the holographic 


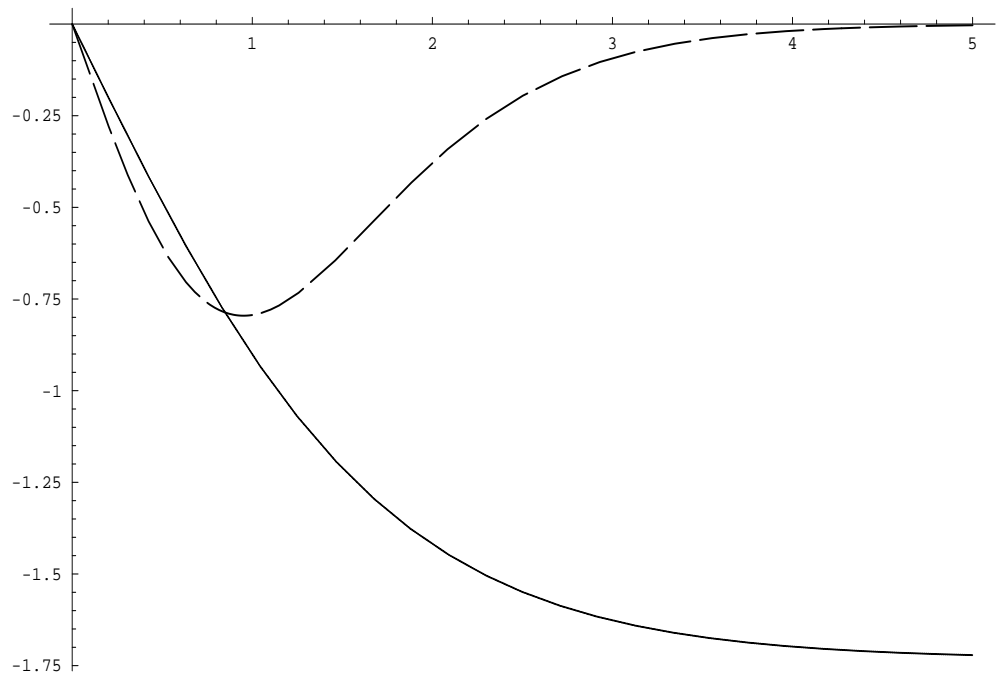

Figure 1: Plot of the holographic (continuous line) and proper (dashed line) beta functions versus the holographic coupling.

beta function. In Fig. 1 the two beta functions are compared for the model of [7], involving the flow to the (confining) pure $\mathrm{N}=1$ super-Yang Mills theory. The holographic beta function tends to a costant in the IR, while the proper beta function better resembles an ordinary beta function. When $c_{\mathrm{IR}}=0$, as in our last example, it is natural to expect that the holographic scheme (23) is still consistent, because, although the holographic beta function tends to a constant, the metric $f$ is zero in the "null" IR theory. This is what our explicit computation of the $T_{\mu \nu}$ correlator shows.

$i v$ ) In the presence of many couplings the formula $\dot{c}=-2 c \beta_{a} \beta_{b} G^{a b}$ does not give all the beta functions separately. Yet, the sum $\beta_{a} \beta_{b} G^{a b}$ is sufficient both to fix $h_{*}$ and to identify the fixed points. In this sense we may call

$$
\beta_{\mathrm{H}} \equiv-\sqrt{\beta_{a} \beta_{b} G^{a b}}=-\sqrt{-\frac{\dot{c}}{2 c}}
$$

the holographic beta function, so that $\dot{c}=-2 c \beta_{\mathrm{H}}^{2}$. The proper beta function is instead $\beta_{\mathrm{P}}=\beta_{\mathrm{H}} \sqrt{2 c}$, so that $\dot{c}=-\beta_{\mathrm{P}}^{2}$.

$v$ ) With obvious changes, various formulas above apply for the $a$-function of [2] in the general case $c \neq a$. Indeed, the relationship between the critical exponent $h_{*}$ and the $a$-function does not require inputs from the AdS/CFT correspondence and holds purely in quantum field theory. This generalization is straightforward and left to the reader.

vi) In refs. [10, explicit formulas for the two-point function of minimally-coupled, massless scalars in the Coulomb branch of $\mathrm{N}=4$ supersymmetric gauge theory are given. From those formulas, one can extract a $c$-function using the same techniques described in 


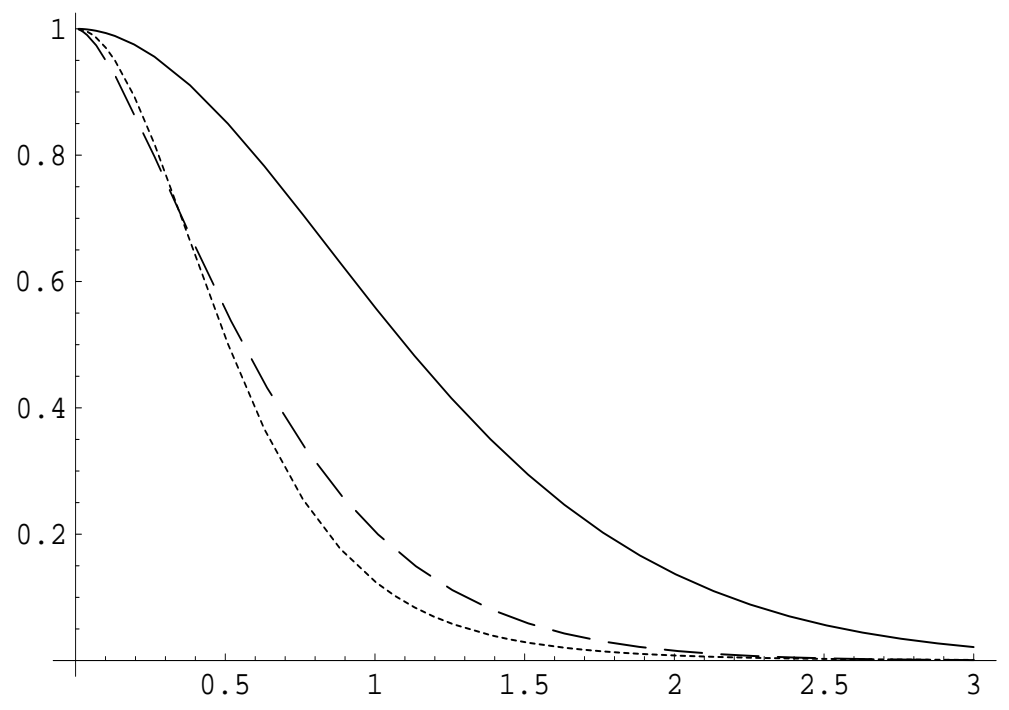

Figure 2: Plot of $8 c / N^{2}$ vs $x$, for different cases. In the case of the flow to $\mathrm{N}=1 \mathrm{SYM}$, the canonical $c$ (eq. (19)) is given by the dashed line, and the holographic $c$ (eq. (22)) is given by the dotted line. Finally, in the case of $\mathrm{N}=4$ Coulomb branch, the canonical $c$-function (eq. (27)) is given by the continuous line.

this paper. As an example we now briefly discuss the case of a a 4-dimensional distribution of branes, giving rise to the two-point function described in eq. (25) of ref. [10]. Following the same steps that led us to eq. (19) we find the central function:

$$
c(x)=\frac{N^{2}}{4} \sum_{n=2}^{\infty}(2 n-1) \sqrt{n^{2}-n}|x|^{3} K_{1}\left(2 \sqrt{n^{2}-n}|x|\right) .
$$

This function is also positive and monotonic as shown in Figure 2.

The holographic scheme is natural and simple. Other schemes and definitions for $c$-functions are less natural from the point of view of the AdS/CFT correspondence, but still have great interest in their own and give results for $c=a$ theories that share many properties with $2 \mathrm{~d}$ conformal field theories. In particular, we considered the definition of a $c$-function from the two-point function of the stress-energy tensor. We computed such a $c(x)$ for a particular supersymmetric flow. The fact that it is monotonic is a highly non-trivial check of the AdS/CFT correspondence as well as of the fact that supergravity solutions may be interpreted as quantum field theory RG flows. Notice that the particular solution used in the computation is singular in the IR (as it happens for all the cases where analytical computations of two-point functions can be performed). Nevertheless, we obtained a sensible result, which indicates that the basic physical properties of such solutions are not completely spoiled by the IR singularity.

We conclude by mentioning some possible extensions of this work that we find partic- 
ularly interesting. 1) To compute the two-point function for a holographic flow between CFTs, as the one connecting the $\mathrm{N}=4$ theory to an IR $\mathrm{N}=1 \mathrm{CFT}$, discussed in ref. 99. 2) To prove in full generality that the canonical c-function is always monotonic, as it happens for the holographic $c$-function. 3) To generalize formula (8) to the canonical $c$-function.

\section{Acknowledgments}

We would like to thank D. Z. Freedman, M. Petrini and A. Starinets for useful discussions. L.G. and A. Z. are partially supported by INFN and MURST, and by the European Commission TMR program ERBFMRX-CT96-0045, wherein they are associated to the University of Torino. M.P. is supported in part by NSF grant no. PHY-9722083.

\section{References}

[1] D. Anselmi, D.Z. Freedman, M.T. Grisaru, A.A. Johansen, Nucl. Phys. B526 (1998) 543, hep-th/9708042.

[2] D. Anselmi, Ann. Phys. (NY) 276 (1999) 361, hep-th/9903059.

[3] D. Anselmi, hep-th/9908014. Phys. Lett. B in press.

[4] M. Henningson and K. Skenderis, JHEP 9807 (1998) 023, hep-th/9810254.

[5] L. Girardello, M. Petrini, M. Porrati and A. Zaffaroni, JHEP 9812 (1998) 022, hepth/9810126.

[6] L. Girardello, M. Petrini, M. Porrati and A. Zaffaroni, JHEP 9905 (1999) 026, hepth/9903026.

[7] L. Girardello, M. Petrini, M. Porrati and A. Zaffaroni, hep-th/9909047, to appear in Nucl. Phys. B.

[8] J. Distler and F. Zamora, Adv. Theor. Math. Phys. 2 (1999) 1405, hep-th/9810206; hep-th/9911040.

[9] D.Z. Freedman, S.S. Gubser, K. Pilch and N.P. Warner, hep-th/9904017.

[10] D.Z. Freedman, S.S. Gubser, K. Pilch and N.P. Warner, hep-th/9906194.

[11] D. Anselmi, M. Grisaru and A.A. Johansen, Nucl.Phys. B491 (1997) 221, hepth/9601023. 
[12] D. Anselmi, JHEP 9805 (1998) 005, hep-th/9702056.

[13] J. de Boer, E. Verlinde and H. Verlinde, hep-th/9912012.

[14] M. Abramowitz and I. Stegun, Handbook of Mathematical Functions, (Dover Press, New York, 1972).

[15] S.S. Gubser, I.R. Klebanov and A.M. Polyakov, Phys. Lett. B428 (1998) 105, hepth/9802109.

[16] E. Witten, Adv. Theor. Math. Phys. 2 (1998) 253, hep-th/9802150. 